\title{
Current-Voltage Characteristics of ITO/p-Si and ITO/n-Si Contact Interfaces
}

\author{
Gopal G. Pethuraja $^{1,2}$, Roger E. Welser ${ }^{1,2}$, Ashok K. Sood ${ }^{1,2}$, Changwoo Lee ${ }^{3}$, Nicholas J. Alexander ${ }^{3}$, \\ Harry Efstathiadis ${ }^{3}$, Pradeep Haldar ${ }^{3}$, Jennifer L. Harvey ${ }^{4}$ \\ ${ }^{1}$ Magnolia Solar Incorporated, Woburn, USA \\ ${ }^{2}$ Magnolia Solar Incorporated, Albany, USA \\ ${ }^{3}$ College of Nanoscale Science and Engineering, Albany, USA \\ ${ }^{4}$ New York State Energy Research and Development Authority, Albany, USA \\ Email: gpethuraja@magnoliasolar.com
}

Received March 2, 2012; revised March 31, 2012; accepted April 24, 2012

\begin{abstract}
We investigated the electrical contact characteristics of indium tin oxide (ITO)/doped hydrogenated amorphous silicon (a-Si:H) junctions. For efficient collection of photo-generated carriers, photovoltaic and photodetector devices require good ohmic contacts with transparent electrodes. The amorphous-Si thin films were sputter deposited on ITO coated glass substrates. As-deposited p-type a-Si:H on ITO formed nearly ohmic type contacts and further annealing did not improve the contact characteristics. On the other hand, as-deposited n-type a-Si:H on ITO formed an ohmic contact, while further annealing resulted in a Schottky type contact. The ITO contact with p-type silicon semiconductor is a robust ohmic contact for Si based optoelectronic devices.
\end{abstract}

Keywords: Sputtered Amorphous Silicon; Electrical Contact Characteristics; ITO/Si Contact

\section{Introduction}

Indium tin oxide (ITO) layers are frequently used as front contacts in thin film optoelectronic devices such as solar cells, light emitting diodes, laser diodes, and photodectors due to their high conductivity and transparency in the visible range of the solar spectrum [1,2]. In the case of thin film solar cells, the ITO contact is necessary to allow photons to reach the absorber layer and improve the photo-generated carrier collection [3]. Solar cells based on amorphous silicon consist of several layers of different chemical composition and hence different optical and electronic properties [4]. The growth of amorphous silicon on transparent conductive oxides such as ITO will undergo an interfacial reaction and the initial stage of growth will be different from the deposition of bulk materials [5]. The reduction of tin oxide $\left(\mathrm{SnO}_{2}\right)$ in hydrogen plasma may influence the contact resistance of the ITO/Si junction [6]. In order to achieve high performance devices, it is required to have ohmic contact with low contact resistance.

The deposition of thin film solar cell structures on low cost and flexible substrates like plastic foil has necessitated the deposition of the thin films at relatively low temperature. Various low temperature schemes have been adapted to prepare a-Si:H films. They include low-power radio frequency (RF), direct current, electron cyclotron resonance and very high frequency - plasma-enhanced chemical vapor deposition $[7,8]$. However, these methods yield low growth rate. For inexpensive photovoltaic (PV) devices, high growth rate is required for depositing 0.5 $\mu \mathrm{m}$ thick absorber layers. Sputtering is a low cost, high deposition rate method that yields high purity films. Sputter deposited ITO on Si wafers or on Si films has been studied by various research groups [5,9], but there is not much work on the contact characteristics of the junction between ITO and sputter deposited amorphous Si films. In this article, we report on the electrical contact properties of ITO on p- and n-type a-Si:H films. Our results shows that the ITO/p-type a-Si:H contact interface is better for silicon based optoelectronic device applications.

\section{Experimental Procedure}

P-type and n-type Si:H films were deposited by a AJA Orion 1800F RF magnetron Sputter System (13.56 MHz) equipped with a load lock. The sputter deposition were carried out in an ultra pure argon $(\mathrm{Ar})+$ hydrogen $\left(\mathrm{H}_{2}\right)$ atmosphere, on commercially procured fluorinated indium tin oxide coated glass substrates. Four-inch diameter borondoped Si and phosphorus-doped Si targets were used for depositing n-type Si and p-type Si films respectively. The substrates were cleaned using acetone, methanol, isopropanol and de-ionized water before loading into the depo- 
sition chamber for plasma cleaning before deposition of the Si film. The system base vacuum was approximately $1 \times 10^{-7}$ Torr, while the process pressure was $3 \mathrm{~m}$ Torr. A fixed RF power of $150 \mathrm{~W}$ was used for a $5 \mathrm{~cm}$ distance between the target and the sample. The substrates were kept at $200^{\circ} \mathrm{C}$ during film deposition. The $\mathrm{Ar}$ and $\mathrm{H}_{2}$ flow rate was maintained at 10 and $1 \mathrm{sccm}$ respectively. Figure 1 shows the schematic of the AJA Orion $1800 \mathrm{~F}$ RF/DC Sputter deposition system used for the sample preparation. For contact resistance studies, $\mathrm{Al} / \mathrm{Si} / \mathrm{ITO}$ test structures were formed by depositing $\mathrm{Al}$ on $\mathrm{Si} / \mathrm{ITO}$ samples. Post deposition annealing of test structures were carried out at $300^{\circ} \mathrm{C}$ in vacuum.

Raman spectroscopy of the films was carried out using a Renishaw inVia confocal Raman spectrometer equipped with a research-grade Leica microscope, $20 \times$ objective (numerical aperture of 0.40 ), and WiRE 2.0 software. A $785 \mathrm{~nm}$ laser light was utilized for excitation. The laser power on the sample was approximately $115 \mathrm{~mW}$.

Cross-sectional transmission electron microscopy (TEM) specimens were prepared from the ITO/p-type a-Si:H/Al sample using a conventional ex-situ lift-out technique in a FEI Nova Nanolab 600 Dualbeam. The specimens were characterized by using a JEOL 2010F operated at $200 \mathrm{kV}$, equipped with an EDAX Si-Li energy dispersive X-ray spectroscopy detector. Currentvoltage $(I-V)$ measurements were performed for ITO/p-Si and ITO/n-Si hetero-structures at room temperature using a Keithely 2400 SourceMeter. The contacts were made using Signatone 1160 series probe station.

\section{Results and Discussion}

All deposited films in this work were thoroughly characterized by Raman spectroscopy and TEM. Raman spectroscopy observation indicated that as-deposited films are amorphous silicon. Figure 2 shows a Raman spectrum taken on as-deposited $\mathrm{p}$-Si films. Si-Si vibrational peaks can be seen around 160 and $480 \mathrm{~cm}^{-1}$. The Raman spectrum of amorphous silicon consists of two distinct bands,

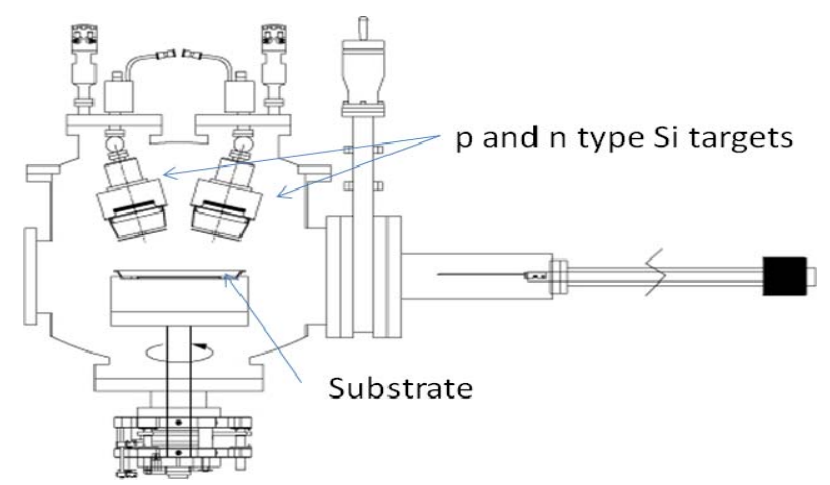

Figure 1. Schematic of the AJA Orion 1800F RF/DC Sputter deposition system used to deposit thin films of $p$ - and n-type a-Si:H on ITO/Glass substrates.

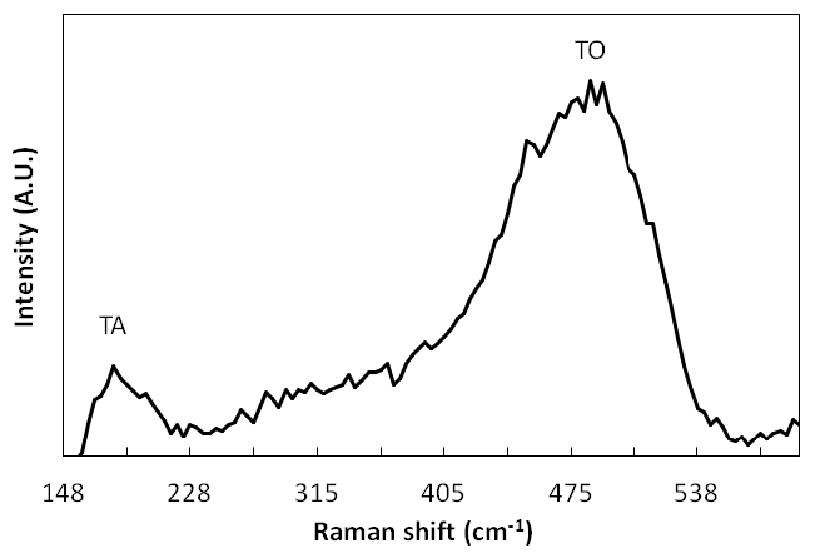

Figure 2. Raman spectroscopy scan of as-deposited p-type a-Si:H film. The peaks at 160 and $480 \mathrm{~cm}^{-1}$ correspond to amorphous Si-Si transverse acoustic (TA) and transverse optic (TO) vibrational modes, respectively.

near $160 \mathrm{~cm}^{-1}$ and $480 \mathrm{~cm}^{-1}$, associated with transverse acoustic (TA) and transverse optic (TO) vibrational modes, respectively [10]. The appearance of the TA-like phonon mode is associated with the network formation of a-Si or the onset of layer growth. Similar results were observed for $\mathrm{n}$ type a-Si:H films. Hence, the sputter deposited silicon films processed for this study are in an amorphous phase.

TEM analysis also confirms amorphous nature of the as-deposited p-type and n-type Si films and the as-deposited films are around $50 \mathrm{~nm}$ thick. Figure 3 shows crosssectional TEM micrograph of ITO/p-Si/Al interface. No grains are seen in the Si layer, confirming the Raman spectroscopy result of an amorphous phase. It also shows that the interface between ITO and $\mathrm{Si}$ is rough. The p-type $\mathrm{Si}$ layer thickness measured from this micrograph is around $50 \mathrm{~nm}$.

Figure 4 shows the currentvoltage characteristics of ITO/p-Si junctions before and after annealing. The $I-V$ characteristics show nearly linear behavior except for a slight deviation in the range of -0.5 to 0.5 volts. The contact resistance at voltages above $0.5 \mathrm{~V}$ is around 15 ohms, while the resistance in the -0.5 to $0.5 \mathrm{~V}$ range is around $30 \mathrm{ohms}$. This indicates that there is an interfacial Schottky barrier that requires 0.5 volt to overcome the barrier. The Schottky contact barrier height at the metal and p-type semiconductor interface is given below:

$$
\varphi_{B}=E_{g}-\left(\varphi_{M}-\chi_{S}\right)
$$

where $E_{g}$ is the bandgap of the semiconductor, $\varphi_{M}$ is the metal work function and $\chi_{S}$ is the semiconductor electron affinity. The work function for ITO is $4.7 \mathrm{eV}$, while the electron affinity for p-type a-Si:H is roughly $3.4 \mathrm{eV}$ and it band gap is $1.8 \mathrm{eV}$. Hence, the calculated barrier height at the ITO/p-Si interface is around $0.5 \mathrm{eV}$. In addition, interface mixing also influences the contact properties. It is known, for instance that the sputter deposition of ITO on 


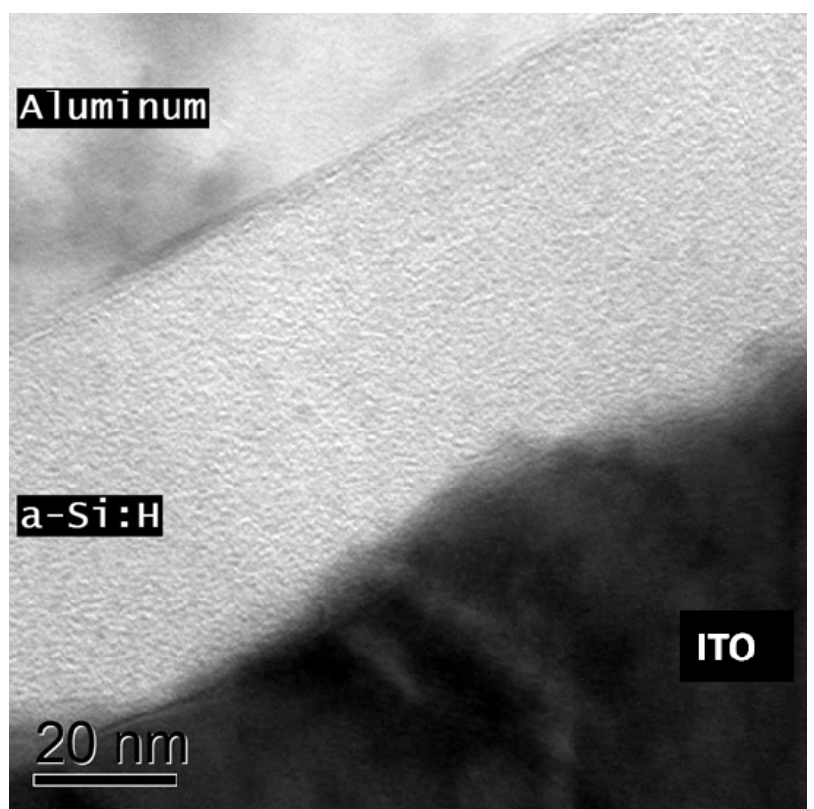

Figure 3. Transmission electron micrograph of the Glass/ ITO/p-type Si:H/Al sample. The silicon film has an amorphous structure and the layer thickness is around $50 \mathrm{~nm}$.

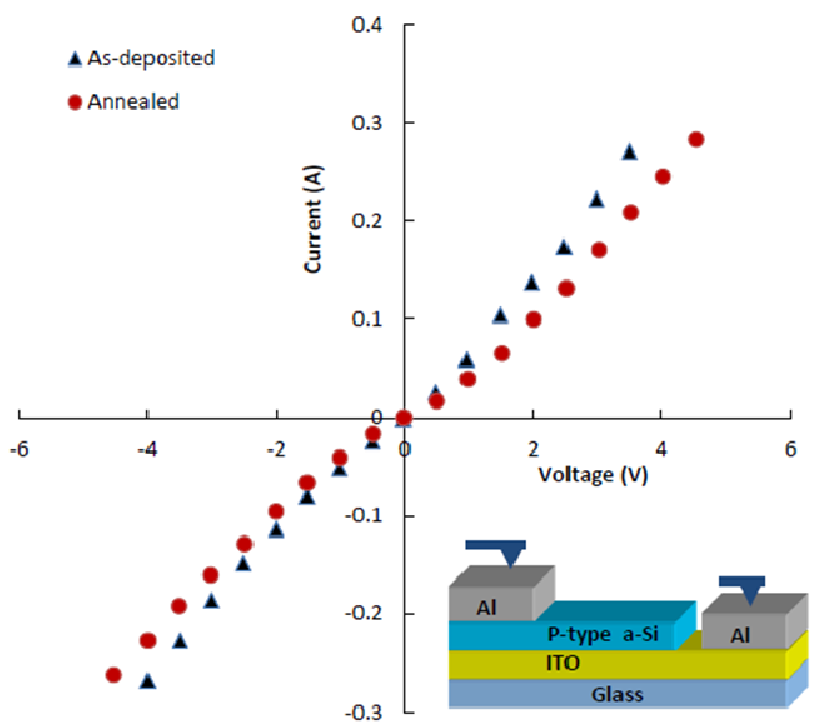

Figure 4. Current-voltage characteristics of ITO/p-type a-Si:H thin film junctions after deposition and annealing. The inset shows the schematic of the sample used for the measurement. The nearly liner current-voltage curve indicates the formation of ohmic contact between ITO and p-type silicon.

Si can create an intermixed damaged layer to a depth of 2 - $3 \mathrm{~nm}$ even at room temperature [11]. In our case, during sputter deposition of Si on ITO, energetic Si atoms deposited on ITO can lead to formation of an interfacial oxide layer. The free energy of formation for $\mathrm{SiO}_{2}, \mathrm{In}_{2} \mathrm{O}_{3}$ and $\mathrm{SnO}_{2}$ are $-193,-207$ and $-124 \mathrm{kcal} / \mathrm{mole}$ respectively [12]. Thus, $\mathrm{In}_{2} \mathrm{O}_{3}$ and indium rich oxides may be expected to yield a stable interface with silicon, but the diffusion of oxygen from $\mathrm{SnO}_{2}$ into the silicon layer may form an interfacial oxide layer. On the other hand, ITO substrates with an indium oxide rich top surface layer could minimize the formation of an interfacial oxide layer. Thus, the $I-V$ behavior of the as-deposited structure can be attributed to the presence of the interfacial oxide layer. Annealing does not improve the $I-V$ curves. It is also known that during PECVD growth of $\mathrm{Si}$, the hydrogen plasma reduces the ITO surface layer that leads to metallic interface layer. But in sputtered Si deposition, the hydrogen plasma interacts with $\mathrm{Si}$ at the target, not at the substrate. The high energetic neutral $\mathrm{Si}$ atoms that deposit on ITO are more favorable to form a thin interfacial oxide layer.

Figure 5 shows the current-voltage characteristics of ITO/n-Si junction before and after annealing. The asdeposited junction shows a linear current-voltage characteristics indicating ohmic contact with a contact resistance of approximately $9 \mathrm{ohms}$ at the junction. After annealing, non-linear, Schottky diode like current-voltage characteristics with a contact resistance of approximately $100 \mathrm{M}$ ohms is observed. N-type silicon layer is expected to form a Schottky contact with ITO due to its work function. The Schottky barrier height at the metal and n-type semiconductor interface is given below:

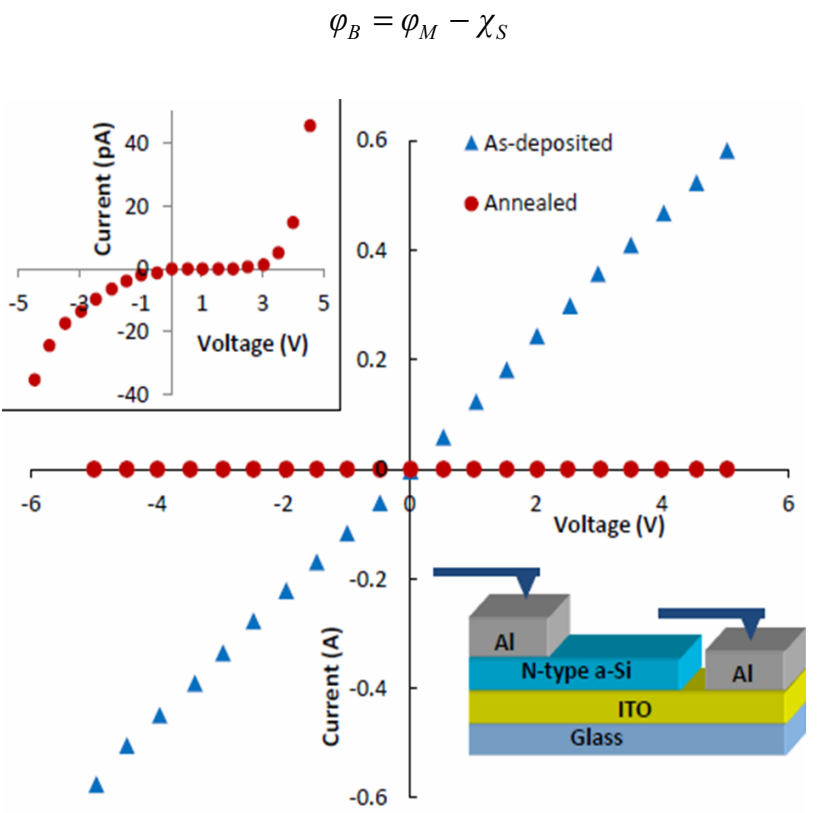

Figure 5. Current-voltage characteristics of ITO/n-type a-Si:H thin film junctions after deposition and annealing. The as-deposited n-type Si film shows ohmic contact with ITO. After annealing, the contact deteriorates to a Schottky type contact with increased contact resistance. The top inset shows the current-voltage characteristics of the annealed sample. The bottom inset shows the schematic of the sample used for the measurement. 
where $\varphi_{M}$ is the metal work function and $\chi_{S}$ is the semiconductor electron affinity. The work function for ITO is $4.7 \mathrm{eV}$ and the electron affinity for n-type a-Si:H is approximately $3.8 \mathrm{eV}$. Hence the barrier height at the ITO/ $\mathrm{n}$-Si interface is approximately $0.9 \mathrm{eV}$. The ohmic contact behavior of as-deposited ITO/n-Si interface is due to formation of interface defects. During post deposition thermal treatment, these defects are annealed out and the formation of an interfacial oxide takes place [13]. The ITO serves as an oxygen source for the formation of the interfacial oxide. Hence, the annealed ITO/n-Si junction shows Schottky type contact behavior with higher resistance compare to the as-deposited interface.

\section{Summary}

Amorphous Si films were fabricated by sputter deposition on ITO/glass substrates in an ultra high vacuum chamber. The electrical contact properties of ITO/p-Si and ITO/n-Si were characterized. P-type silicon film on ITO forms a nearly ohmic type contact, while n-type silicon on ITO forms a Schottky type contact. P-type $\mathrm{Si} / \mathrm{ITO}$ contacts are a promising, robust ohmic contact for silicon-based optoelectronic devices.

\section{Acknowledgements}

The authors would like to thank NYSERDA for supporting our work on thin-film silicon materials for solar cell applications.

\section{REFERENCES}

[1] Y. C. Lin, S. J. Chang, Y. K. Su, T. Y. Tsai, C. S. Chang, S. C. Shei, S. J. Hsu, C. H. Liu, U. H. Liaw, S. C. Chen and B. R. Huang, "Nitride-Based Light-Emitting Diodes with Ni/ITO P-Type Ohmic Contacts," IEEE Photonics Technology Letters, Vol. 14, No. 12, 2002, pp. 1668-1670. doi:10.1109/LPT.2002.804649

[2] D. W. Kim, Y. J. Sung, J. W. Park and G. Y. Yeom, "A Study of Transparent Indium Tin Oxide (ITO) Contact to p-GaN," Thin Solid Films, Vol. 398-399, 2001, pp. 87-92.

[3] T. J. Coutts, D. L. Young and X. N. Li, "Characterization of Transparent Conducting Oxides," MRS Bulletin, Vol. 25, No. 8, 2000, pp. 58-65. doi:10.1557/mrs2000.152

[4] H. Stiebig, F. Siebke, W. Beyer, C. Beneking, B. Rech and H. Wagner, "Interfaces in a-Si: H Solar Cell Struc- tures," Solar Energy Materials and Solar Cells, Vol. 48, No. 1-4, 1997, pp. 351-363.

doi:10.1016/S0927-0248(97)00147-5

[5] E. V. Monakhov, R. Balasundaraprabhu, N. Muthukumarasamy and B. G. Svensson, "Electronic Properties of the Interface between $\mathrm{Si}$ and Sputter Deposited IndiumTin Oxide," Materials Science and Engineering: B, Vol. 159-160, 2009, pp. 314-317. doi:10.1016/j.mseb.2008.11.023

[6] K. S. Son, D. L. Choi, H. N. Lee and W. G. Lee, "The Interfacial Reaction between ITO and Silicon Nitride Deposited by PECVD in Fringe Field Switching Device," Current Applied Physics, Vol. 2, No. 3, 2002, pp. 229-232. doi:10.1016/S1567-1739(02)00092-5

[7] E. Maruyama, S. Okamoto, A. Terakawa, W. Shinohara, M. Tanaka and S. Kiyama, "Towards Stabilized 10\% Efficiency of Large-Area $\left(>5000 \mathrm{~cm}^{2}\right)$ a-Si/a-SiGe Tandem Solar Cells Using High-Rate Deposition," Solar Energy Materials and Solar Cells, Vol. 71, No. 1-4, 2002, pp. 339349. doi:10.1016/S0927-0248(02)00093-4

[8] P. Wickboldt, D. Pang, W. Paul, J. H. Chen, F. Zhong, C. C. Chen, J. D. Cohen and D. L. Williamson, "High Performance Glow Discharge a-Si $i_{1-x} \mathrm{Ge}_{x}: \mathrm{H}$ of Large $x$," Journal of Applied Physics, Vol. 81, No. 9, 1997, pp. 62526267. doi:10.1063/1.364413

[9] M. Kubon, E. Behmer, F. Siebke, B. Rech, C. Beneking and $\mathrm{H}$. Wagner, "Solution of the $\mathrm{ZnO} / \mathrm{p}$ Contact Problem in a-Si:H Solar Cells," Solar Energy Materials and Solar Cells, Vol. 41-42, 1996, pp. 485-492. doi:10.1016/0927-0248(95)00126-3

[10] B. P. Swain, "The Analysis of Carbon Bonding Environment in HWCVD Deposited a-SiC:H Films by XPS and Raman Spectroscopy," Surface and Coatings Technology, Vol. 201, No. 3-4, 2006, pp. 1589-1593. doi:10.1016/j.surfcoat.2006.02.029

[11] M. A. Martinez, M. T. Gutierrez and C. Maffiotte, "Chemical Changes of ITO/p and $\mathrm{ZnO} / \mathrm{p}$ Interfaces as a Function of Deposition Parameters," Surface and Coatings Technology, Vol. 110, No. 1-2, 1998, pp. 68-72. doi:10.1016/S0257-8972(98)00673-2

[12] J. B. Dubow, D. E. Burk and J. R. Sites, "Efficient Photovoltaic Heterojunctions of Indium Tin Oxides on Silicon," Applied Physics Letters, Vol. 29, No. 8, 1976, pp. 494-496. doi:10.1063/1.89135

[13] C. W. Ow-Yang, Y. Shigesato and D. C. Paine, "Interface Stability of an Indium Tin Oxide Thin Film Deposited on $\mathrm{Si}$ and $\mathrm{Si}_{0.85} \mathrm{Ge}_{0.15}$," Journal of Applied Physics, Vol. 88, No. 6, 2000, pp. 3717-3724. doi:10.1063/1.1288694 